\title{
Structure Analysis of Cation Selective Cr-goethite as Protective Rust of Weathering Steel
}

\author{
Hiroyuki Konishi ${ }^{1}$, Masato Yamashita ${ }^{2}$, Hitoshi Uchida ${ }^{2}$ and Jun'ichiro Mizuki ${ }^{1}$ \\ ${ }^{1}$ Synchrotron Radiation Research Center, Kansai Research Establishment, Japan Atomic Energy Research Institute, \\ Sayo-gun, Hyogo 679-5143, Japan \\ ${ }^{2}$ Division of Mechanical Engineering, Graduate School of Engineering, University of Hyogo, Himeji, Hyogo 671-2201, Japan
}

We have performed extended X-ray absorption fine structure (EXAFS) analysis for artificial Cr-goethite to elucidate the local structure around $\mathrm{Cr}$ in Cr-goethite. The spectra were obtained using synchrotron radiation X-rays at the Photon Factory in Tsukuba. The first shell contributions were isolated by Fourier filtering the EXAFS data, and the inverse Fourier transformed single-shell data were analyzed using a curve fitting method. The results show that $\mathrm{Cr}$ is coordinated with $(7 \pm 1) \mathrm{O}^{2-}$ ions. The protective characteristics of the $\mathrm{Cr}$-goethite protective rust layer on weathering steel can be interpreted in terms of the $\mathrm{O}^{2-}$ coordination around $\mathrm{Cr}^{3+}$ resulting in the creation of negative fixed charge in the Cr-goethite particles.

(Received November 16, 2004; Accepted December 20, 2004)

Keywords: extended X-ray absorption fine structure, chromium $K$ absorption edge, protective rust layer, Cr-goethite, cation selectivity, weathering steel, atmospheric corrosion, corrosion resistance, synchrotron radiation

\section{Introduction}

The study of the rust layer formed on steel and its control lead to an improvement in the performance of steel. A rust layer formed on a low-alloy steel surface is generally able to impede the penetration of corrosives from an atmosphere. This ability of the so-called 'protective' rust layer enables the atmospheric corrosion resistance of the steel. It is wellknown that weathering steel, which contains small amounts of $\mathrm{Cr}, \mathrm{P}$ and $\mathrm{Cu}$, possesses high atmospheric corrosion resistance because of the strong protective ability of the rust layer formed on the steel for impeding the penetration of corrosives.

Various spectroscopic studies such as X-ray diffraction, ${ }^{1-3)}$ Raman, $^{4,5)}$ infrared ${ }^{6,7)}$ and Mössbauer ${ }^{8-10)}$ spectroscopies has been carried out to understand the protective ability of the rust layer in terms of its structure. These studies revealed that the protective rust layer of weathering steel has a doublelayer structure and its inner layer is mainly composed of ultrafine crystals of $\alpha$-FeOOH containing small amounts of $\mathrm{Cr}$. This ferric oxyhydroxide is called $\mathrm{Cr}$-goethite. The protective ability of the rust layer is considered to be derived from the densely packed texture of the inner layer. ${ }^{11)}$

Additionally, Miyuki et al. found that $\mathrm{Cr}$-goethite possesses selectivity against cation penetration depending on $\mathrm{Cr}$ concentration by measuring the membrane potentials of artificial Cr-goethite. ${ }^{12)}$ They pointed out that this cation selectivity results in a high protective ability of the rust layer. However, there has been no study that has attempted to investigate the cation selectivity from the viewpoint of the properties of the structure around $\mathrm{Cr}$.

It is known that the goethite crystal structure consists of double chains of $\mathrm{FeO}_{3}(\mathrm{OH})_{3}$ octahedra formed by edge sharing. ${ }^{13)}$ These chains are linked to adjacent double chains by corner sharing and the double chains of vacant sites run parallel to the double chains of octahedra in the goethite structure. However, the detailed structure around $\mathrm{Cr}$ in the $\mathrm{Cr}$-goethite was unclear. We have already performed X-ray absorption fine structure (XAFS) studies using synchrotron radiation X-rays to examine the local structural and chemical properties of $\mathrm{Cr}$ ions in atmospheric rust layers of weathering steel ${ }^{14)}$ and $\mathrm{Fe}-5$ mass $\% \mathrm{Cr}$ alloy film. ${ }^{15)}$ The weathering steel was exposed to the industrial atmosphere underneath the girder of a bridge at Yokkaichi, Japan, for 17 years. The $100 \mathrm{~nm}$-thick Fe-Cr alloy film, which was vapor deposited on a $\mathrm{Si}$ single-crystal substrate, was corroded rapidly under a $100 \mu \mathrm{m}$-thick electrolyte film.

From these studies employing atmospheric corrosion products, it was indicated that most $\mathrm{Cr}^{3+}$ ions do not substitute into the $\mathrm{Fe}^{3+}$ site at the center of the $\mathrm{FeO}_{3}(\mathrm{OH})_{3}$ octahedra but form $\mathrm{CrO}_{x}{ }^{3-2 x}$ complex ions with a plural number of $\mathrm{O}^{2-}$ in the rust layer. However, we could not conduct further analysis to obtain the detailed structure of $\mathrm{Cr}$. Because the weathering steel and its rust layer that were used in the previous study contained small amounts of $\mathrm{Mn}$, an additional absorption spectrum above the Mn K-edge $(6539 \mathrm{eV})$ overlapped with the extended X-ray absorption fine structure (EXAFS) spectrum around the $\mathrm{Cr}$ K-edge. Also, the amount of rust obtained by corroding the $\mathrm{Fe}-\mathrm{Cr}$ alloy film was very limited.

In the present study, we employ artificial $\mathrm{Mn}$-free $\mathrm{Cr}$ goethite samples and examine the local structure around $\mathrm{Cr}$ in $\mathrm{Cr}$-goethite by EXAFS analysis using synchrotron radiation X-rays.

\section{Experimental}

\subsection{Sample preparation}

The preparation method used for our powder samples has been described in the previous papers. ${ }^{11,12)} \mathrm{Cr}$-goethite powder samples with 1.5, 3.0 and 10 mass $\% \mathrm{Cr}$ were prepared artificially. Pure goethite powder was also synthesized by a similar method. Structure identification was made by X-ray diffraction analysis using synchrotron radiation X-rays at BL09XU in SPring-8. All the diffraction peaks obtained can be assigned to the goethite structure reported in 


\section{JCPDS290713. ${ }^{16)}$}

The powder samples were mixed with boron nitride $(\mathrm{BN})$ powder and were compressed into tablets to obtain homogeneous samples with suitable absorption coefficients for EXAFS measurements in the transmission mode.

\subsection{EXAFS experiments}

The EXAFS spectra of Cr-goethite and pure goethite were obtained around the $\mathrm{Cr} \mathrm{K}$-edge and $\mathrm{Fe} \mathrm{K}$-edge X-ray energies, respectively, at BL27B in the Photon Factory (PF) of the High Energy Accelerator Research Organization (KEK) in Tsukuba. A double-crystal monochromator equipped with Si (111) flat crystals permits the use of monochromatic X-rays. The elimination of higher harmonics was performed by $50 \%$ detuning of the monochromator. The spectra were obtained in the transmission mode at room temperature. The intensities of the incident and transmitted $\mathrm{X}$-rays were detected with two ion chambers.

\subsection{Data Analysis}

In the present study, REX2000, XAFS analysis software made by RIGAKU with a graphical user interface for Microsoft Windows, was used for EXAFS data analysis.

An X-ray absorption spectrum, i.e., an X-ray absorption coefficient $\mu(E)$ as a function of photon energy, $E$, is calculated by

$$
\mu(E) t=\ln I_{0}(E) / I(E),
$$

where $t$ is the sample thickness, and $I_{0}$ and $I$ are the intensities of the incident and transmitted X-rays, respectively. The EXAFS function $\chi(k)$, where $k$ is the wave number of photoelectrons, should be derived from the absorption spectra after background removal, normalization and $E-k$ conversion. Namely, the EXAFS function is defined by

$$
\chi(k)=\left(\mu(k)-\mu_{0}(k)\right) / \mu_{0}(k) .
$$

The background $\mu_{0}(k)$ is usually obtained by a mathematical smoothing method from experimental data. $k$ is expressed in terms of the mass of a photoelectron, $m$, the photon energy of an X-ray, $E$, the edge energy of the absorbing atom, $E_{0}$, and Planck's constant, $\hbar(=h / 2 \pi)$, giving

$$
k=\left[2 m\left(E-E_{0}\right) / \hbar^{2}\right]^{1 / 2} .
$$

The Fourier transform of $\chi(k)$ provides the radial structure function (RSF) in real space. The peak position in the RSF is ordinarily shifted by $0.02-0.05 \mathrm{~nm}$ in the negative direction from the actual distance between the absorbing and neighboring atoms because the phase shift correction is not taken into account.

A single-shell contribution to the EXAFS oscillation can be isolated by Fourier filtering the EXAFS data. Namely, the single-shell EXAFS oscillation can be obtained by inverse
Fourier transforming the RSF multiplied by a window function. The curve fitting by the least-squares method attempts to best fit the filtered EXAFS oscillation to a plane wave and the single scattering EXAFS formula as

$$
\chi(k)=\frac{N}{k R^{2}} f(k) \exp \left(-2 \sigma^{2} k^{2}\right) \exp (-2 R / \lambda) \sin (2 k R+\phi),
$$

where $N$ is the coordination number, $r$ is the atomic distance, $\sigma$ is the Debye-Waller factor and $\lambda$ is the mean free path of a photoelectron. The photoelectron backscattering factor $f(k)$ and phase shift $\phi$ are obtained from McKale's table ${ }^{17)}$ by the software REX2000.

In general, it is difficult to obtain a precise value of $\lambda$ theoretically. Strictly, $\lambda$ is a function of $\mathrm{k}$. However, its $\mathrm{k}$ dependence is usually neglected, and $\lambda$ is regarded as a constant in the calculation process. Because of the weak dependence of $\lambda$ on the absorbing atom species, we adopted the $\lambda$ obtained from the EXAFS data around the Fe K-edge for pure goethite, the structure of which is well-known. As Fe is positioned at the center of the $\mathrm{FeO}_{3}(\mathrm{OH})_{3}$ octahedron in the goethite structure, the coordination number of $\mathrm{Fe}$ in the pure goethite was fixed to be 6 in the curve fitting calculation for the pure goethite EXAFS data around the Fe K-edge. $\lambda$ was regarded as a fitting parameter in this calculation. The analysis result for pure goethite is listed in Table 1. The obtained value of $\lambda$ was used in the analysis of the EXAFS data around the $\mathrm{Cr} \mathrm{K}$-edge for the artificial Cr-goethite.

For a measure of fitting, the residual (R-factor) is defined by

$$
R=\frac{\sum\left(k^{n} \chi_{\mathrm{obs}}-k^{n} \chi_{\mathrm{calc}}\right)^{2}}{\sum\left(k^{n} \chi_{\mathrm{obs}}\right)^{2}},
$$

where $\mathrm{n}$ is a weighting factor used in Fourier transformation, and subscripts 'obs' and 'calc' denote observed and calculated values, respectively.

\section{Results}

The fundamental features of the obtained spectra are similar to those of spectra obtained in the previous studies on weathering steel and $\mathrm{Fe}-\mathrm{Cr}$ alloy film mentioned in the introduction. Figure 1 shows the $\mathrm{Cr}$ K-edge X-ray absorption spectra of the artificial Cr-Goethite powders. The observed Cr-K X-ray absorption edge was $5.995 \mathrm{eV}$. In addition, a well-known peak at $5985 \mathrm{eV}$ for hexavalent $\mathrm{Cr}^{6+}$ was not observed in the spectra. These results indicate that most of the $\mathrm{Cr}$ ions in the rust layers are trivalent $\mathrm{Cr}^{3+}$.

The pre-edge background of the spectra was fitted with Victreen's formula, and the post-edge background $\mu_{0}$ was determined by the spline smoothing method. The EXAFS spectra weighted by $k^{3}, k^{3} \chi(k)$, after background removal, normalization and $E$ - $k$ conversion are shown in Fig. 2.

Table 1 EXAFS analysis result for artificial pure goethite $(\mathrm{Cr}=0 \%)$. The coordination number $N$ was fixed to be 6 in the calculation process.

\begin{tabular}{lccccc}
\hline & $N$ & $\begin{array}{c}r_{\mathrm{Fe}-\mathrm{O}} \\
\left(10^{-1} \mathrm{~nm}\right)\end{array}$ & $\begin{array}{c}\sigma_{\mathrm{Fe}-\mathrm{O}} \\
\left(10^{-1} \mathrm{~nm}\right)\end{array}$ & $\begin{array}{c}\mathrm{R} \text {-factor } \\
\left(10^{-1} \mathrm{~nm}\right)\end{array}$ & $3.206 \pm 0.415$ \\
\hline Artificial goethite & 6 & $1.992 \pm 0.018$ & $0.107 \pm 0.019$ & 5.430 \\
\hline
\end{tabular}




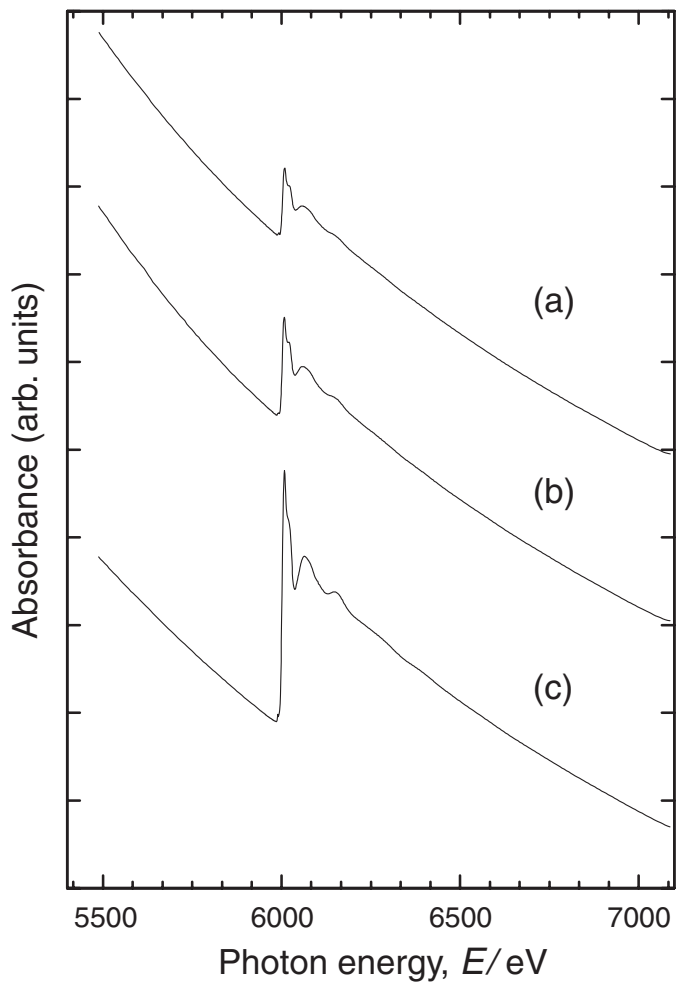

Fig. 1 Raw Cr K-edge X-ray absorption spectra of artificial Cr-goethite of (a) 1.5 mass $\% \mathrm{Cr}$, (b) 3.0 mass $\% \mathrm{Cr}$ and (c) 10 mass $\% \mathrm{Cr}$.

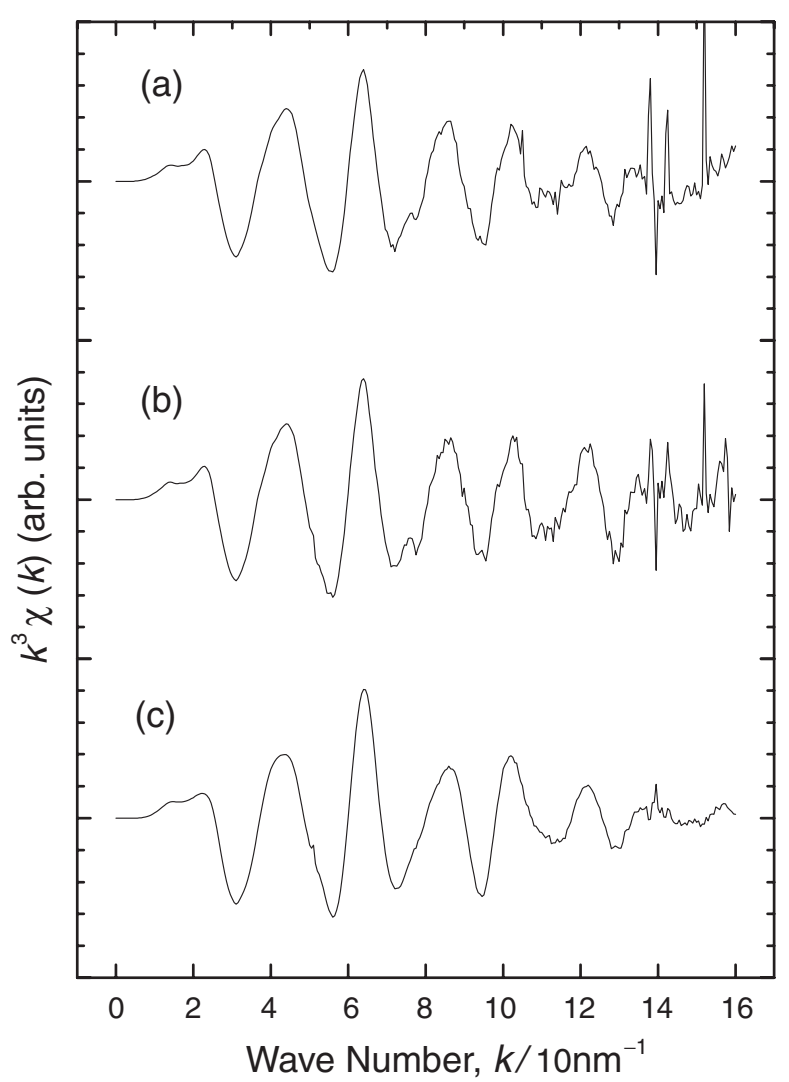

Fig. 2 EXAFS function $k^{3} \chi(k)$ for artificial Cr-goethite of (a) 1.5 mass $\% \mathrm{Cr}$, (b) 3.0 mass $\% \mathrm{Cr}$ and (c) 10 mass $\% \mathrm{Cr}$.

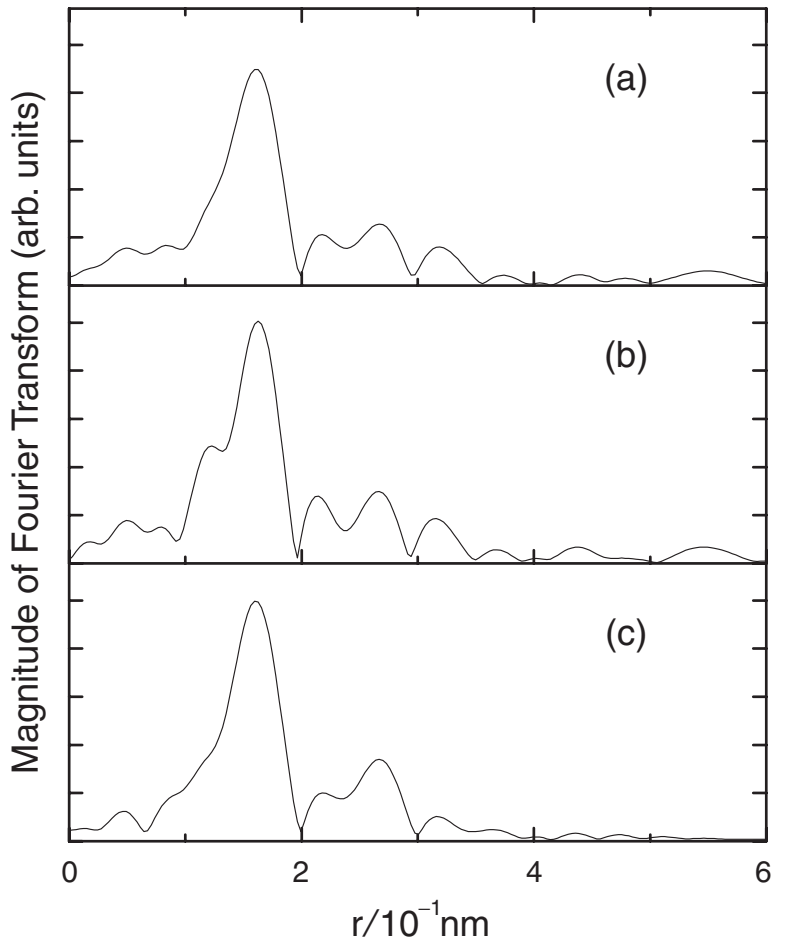

Fig. 3 Fourier transforms of the $k^{3} \chi(k)$ data. (a) 1.5 mass $\% \mathrm{Cr}$-goethite, (b) 3.0 mass $\%$ Cr-goethite, (c) 10 mass $\%$ Cr-goethite.

Fourier transforms of $k^{3} \chi(k)$, i.e., RSFs, over the wave number range of around $0.31 \leqq k / \mathrm{nm} \leqq 1.37$ are shown in Fig. 3. They are not corrected for phase shift. All the RSFs exhibit similar features. The strongest first peak in the RSFs appears at approximately $0.16 \mathrm{~nm}$, thus the corresponding corrected distance is estimated to be $0.16-0.20 \mathrm{~nm}$. Because the ionic radii of $\mathrm{Cr}^{3+}, \mathrm{Fe}^{3+}, \mathrm{Fe}^{2+}$ and $\mathrm{O}^{2-}$ are 0.07, 0.075, 0.069 and $0.13 \mathrm{~nm}$, respectively, the first peak is assigned to $\mathrm{Cr}-\mathrm{O}$ bonds. Therefore, $\mathrm{Cr}^{3+}$ is coordinated with $\mathrm{O}^{2-}$ ions.

As observed in the previous studies, the peaks corresponding to the nearest-neighbor $\mathrm{Cr}-\mathrm{Fe}$ and/or $\mathrm{Cr}-\mathrm{Cr}$ distance do not appear clearly in the RSFs as shown in Fig. 3. Consequently, it was confirmed using the artificial $\mathrm{Cr}$ goethite that there is less correlation above $0.20 \mathrm{~nm}$ around $\mathrm{Cr}^{3+}$ in the Cr-goethite.

Figure 4 shows Fourier-filtered EXAFS oscillations obtained by inverse Fourier transforming the first peaks of the RSFs multiplied by the Hanning function. The curve fitting for the first shell data was performed in order to obtain local structural parameters. No specific structural model of the $\mathrm{CrO}_{x}{ }^{3-2 x}$ complex ion was used in the analysis. However, it was supposed that $\mathrm{Cr}^{3+}$ was coordinated with $\mathrm{O}^{2-}$ with a constant distance.

The photoelectron mean free path $\lambda$ was obtained from the EXAFS data of artificial pure goethite as mentioned previously. This quantity is subject to the error listed in Table 1. The curve fitting for each sample was performed using the minimum, central and maximum values of $\lambda$ in the range of its error.

The best fit structural parameters are listed in Table 2. The $\mathrm{R}$-factors for the successive fittings using different $\lambda$ values were almost identical. Accordingly, it was found that the 
Table 2 EXAFS analysis results for artificial Cr-goethite. Photoelectron mean free path $\lambda$ was fixed at minimum, central and maximum values in the error range obtained for artificial pure goethite.

\begin{tabular}{|c|c|c|c|c|c|}
\hline & $\begin{array}{c}\lambda \\
\left(10^{-1} \mathrm{~nm}\right)\end{array}$ & $N$ & $\begin{array}{c}r_{\mathrm{Cr}-\mathrm{O}} \\
\left(10^{-1} \mathrm{~nm}\right)\end{array}$ & $\begin{array}{c}\sigma_{\mathrm{Cr}-\mathrm{O}} \\
\left(10^{-1} \mathrm{~nm}\right)\end{array}$ & R-factor \\
\hline \multirow[t]{3}{*}{1.5 mass $\%$ Cr-goethite } & 2.8 & $8.457 \pm 0.450$ & $1.999 \pm 0.004$ & $0.056 \pm 0.008$ & 2.216 \\
\hline & 3.2 & $7.023 \pm 0.373$ & $1.999 \pm 0.004$ & $0.056 \pm 0.008$ & 2.214 \\
\hline & 3.6 & $6.090 \pm 0.323$ & $1.999 \pm 0.004$ & $0.056 \pm 0.008$ & 2.216 \\
\hline \multirow[t]{3}{*}{3.0 mass $\%$ Cr-goethite } & 2.8 & $8.361 \pm 0.414$ & $1.999 \pm 0.004$ & $0.049 \pm 0.008$ & 2.423 \\
\hline & 3.2 & $6.933 \pm 0.344$ & $1.999 \pm 0.004$ & $0.049 \pm 0.008$ & 2.424 \\
\hline & 3.6 & $6.013 \pm 0.298$ & $1.999 \pm 0.004$ & $0.049 \pm 0.008$ & 2.426 \\
\hline \multirow[t]{3}{*}{10 mass $\%$ Cr-goethite } & 2.8 & $8.470 \pm 0.411$ & $1.990 \pm 0.004$ & $0.049 \pm 0.008$ & 2.000 \\
\hline & 3.2 & $7.045 \pm 0.341$ & $1.990 \pm 0.004$ & $0.049 \pm 0.008$ & 2.001 \\
\hline & 3.6 & $6.112 \pm 0.296$ & $1.990 \pm 0.004$ & $0.049 \pm 0.008$ & 2.003 \\
\hline
\end{tabular}

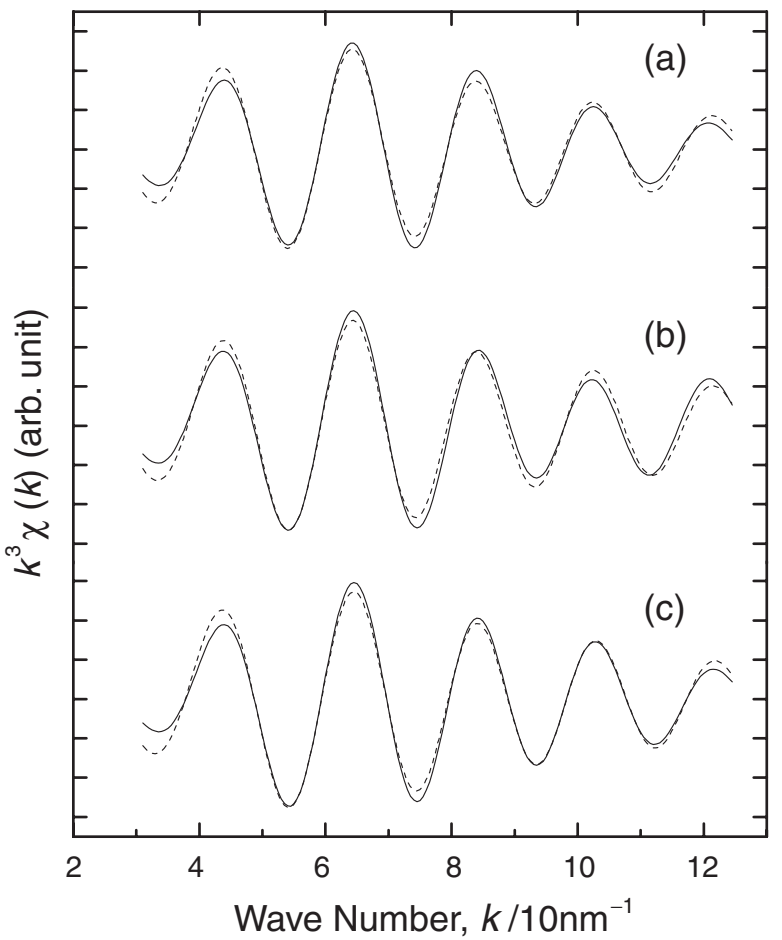

Fig. 4 Inverse Fourier transforms of the first peak of Fourier transformed data shown in Fig. 3. Dashed curves are the best fit results with single distance model. (a) 1.5 mass $\%$ Cr-goethite, (b) 3.0 mass $\%$ Cr-goethite, (c) 10 mass $\%$ Cr-goethite.

coordination number is approximately $(7 \pm 1)$. Its indetermination could be caused by the error of $\lambda$ in the present study.

Another structural parameter determined in this analysis is the $\mathrm{Cr}-\mathrm{O}$ bond length. It is approximately $0.20 \mathrm{~nm}$ in all the fitting results. Additionally, the splitting of the first peak was not found in all the RSFs for different $\mathrm{Cr}$ concentrations. Therefore, the experimental results indicate no local structural change around $\mathrm{Cr}$ in the $\mathrm{Cr}$-goethite related to a change in bond length depending on the $\mathrm{Cr}$ concentration.

\section{Discussion}

The most characteristic protective effect of the $\mathrm{Cr}$-goethite protective rust layer on weathering steel is the cation selectivity which impedes the penetration of aggressive corrosive anions such as $\mathrm{Cl}^{-}$and $\mathrm{SO}_{4}{ }^{2-}$. As mentioned above, we have examined XAFS spectra obtained from the $\mathrm{Cr}$-goethite protective rust layer on weathering steel ${ }^{14)}$ and $\mathrm{Fe}-\mathrm{Cr}$ alloy film. ${ }^{15)}$ The results indicated the formation of the $\mathrm{CrO}_{x}^{3-2 x}$ complex ion in the rust layer.

The same results were obtained in the present study. The peaks above $0.20 \mathrm{~nm}$ of the RSF around the Cr K-edge shown in Fig. 3 were not clearly observed in comparison with those of the RSF around the Fe K-edge obtained previously. ${ }^{14,15)}$ The latter RSF showed two strong peaks around 0.26 and $0.32 \mathrm{~nm}$ corresponding to the nearest-neighbor $\mathrm{Fe}-\mathrm{Fe}$ distance, which is approximately $0.30-0.35 \mathrm{~nm}$, in the goethite structure. Therefore, there is less correlation above $0.20 \mathrm{~nm}$ around $\mathrm{Cr}^{3+}$ in the rust layers. These results indicated that most $\mathrm{Cr}^{3+}$ ions do not substitute into the $\mathrm{Fe}^{3+}$ site at the center of the $\mathrm{FeO}_{3}(\mathrm{OH})_{3}$ octahedra because the $\mathrm{Fe}^{3+}$ site should be strongly correlated to the neighboring $\mathrm{Fe}^{3+}$ sites. On the basis of the results indicating no fixed distance between $\mathrm{Cr}^{3+}$ and neighboring $\mathrm{Cr}^{3+}$ or $\mathrm{Fe}^{3+}$, it has been expected that the $\mathrm{CrO}_{x}{ }^{3-2 x}$ complex ion is located at the surface of and/or grain boundary in the Cr-goethite crystal.

By employing artificial Cr-goethite, we could perform the EXAFS analysis using a curve fitting method for the first time. The curve fitting results of the Fourier filtered EXAFS oscillations for the first shell indicated that the coordination number of $\mathrm{Cr}^{3+}$ is approximately $(7 \pm 1)$ in the $\mathrm{CrO}_{x}{ }^{3-2 x}$ complex ion of the artificial goethite. Because the oxidation numbers of $\mathrm{Cr}$ and $\mathrm{O}$ are considered to be +3 and -2 , respectively, the coordination of $(7 \pm 1) \mathrm{O}^{2-}$ ions results in the negative charge of the $\mathrm{CrO}_{x}{ }^{3-2 x}$ complex ion. This negatively charged ion might create a negative fixed-charge in the $\mathrm{Cr}$-goethite particles. In conclusion, it can be pointed out that the characteristic cation selectivity of Cr-goethite arises from the negatively charged $\mathrm{CrO}_{x}{ }^{3-2 x}$ complex ion in the $\mathrm{Cr}$-goethite protective rust layer.

\section{Conclusion}

The Cr K-edge EXAFS spectra of artificial Cr-goethite with 1.5, 3.0 and 10 mass $\% \mathrm{Cr}$ were measured and analyzed in order to investigate the local structure around $\mathrm{Cr}$ in $\mathrm{Cr}$ goethite as the main component of the protective rust layer on weathering steel. The result of the analysis shows that the number of nearest-neighbor $\mathrm{O}^{2-}$ ions around $\mathrm{Cr}^{3+}$ is $(7 \pm 1)$ 
within the error of the photoelectron mean free path. This indicates that the $\mathrm{CrO}_{x}{ }^{3-2 x}$ complex ion has a negative charge. Accordingly, the $\mathrm{CrO}_{x}{ }^{3-2 x}$ complex ion is considered to provide a negative fixed-charge to the Cr-goethite crystal. The cation selectivity of $\mathrm{Cr}$-goethite can be interpreted in terms of this $\mathrm{O}^{2-}$ coordination around $\mathrm{Cr}^{3+}$. The rust layer containing $\mathrm{Cr}$-goethite shows a protective ability against corrosives having a negative charge such as $\mathrm{SO}_{4}{ }^{2-}$ and $\mathrm{Cl}^{-}$ because of this property.

\section{REFERENCES}

1) T. Misawa, K. Asami, K. Hashimoto and S. Shimodaira: Corros. Sci. 14 (1974) 279-289.

2) M. Yamashita, H. Miyuki, Y. Matsuda, H. Nagano and T. Misawa: Corros. Sci. 36 (1994) 283-299.

3) M. Yamashita and H. Uchida: Hyperfine Interactions 139/140 (2002) 153-166.

4) H. Kihira, S. Ito and T. Murata: Corros. Sci. 31 (1990) 383-388.

5) M. Yamashita, K. Asami, T. Ishikawa, T. Ohtsuka, H. Tamura and T. Misawa: Corrosion Engineering 50 (2001) 733-750.

6) T. Misawa, T. Kyuno, W. Suetaka and S. Shimodaira: Corros. Sci. 11
(1971) 35-48.

7) J. Keiser, C. Brown and R. H. Heidersbach: J. Electrochem. Soc. 129 (1982) 2686-2689.

8) D. C. Cook, R. Balasubramanian, S. J. Oh and M. Yamashita: Hyperfine Interactions 122 (1999) 59-70.

9) M. Yamashita, T. Misawa, H. E. Townsend and D. C. Cook: J. Japan Inst. Metals 64 (2000) 77-78.

10) T. Kamimura, S. Nasu, T. Tazaki, K. Kuzushita and S. Morimoto: Mater. Trans. 43 (2002) 694-703.

11) M. Yamashita, H. Miyuki, H. Nagano and T. Misawa: Proc. 13th Int. Corros. Congress (Melbourne, 1996) 258-264.

12) H. Miyuki, M. Yamashita, M. Fujiwara and T. Misawa: Zairyo-toKankyo 47 (1998) 186-192.

13) See, for example, R. M. Cornell and V. Schwertmann: The Iron Oxides, (WILEY-VCH Verlag GmbH \& Co. KGaA, Weinheim, 2003) 2nd ed., $14-18$.

14) M. Yamashita, H. Konishi, J. Mizuki and H. Uchida: Mater. Trans. 45 (2004) 1920-1924.

15) M. Yamashita, H. Konishi, J. Mizuki and H. Uchida: Corros. Sci. 45 (2003) 381-394.

16) JCPDS, International Centre for Diffraction Data, Newtown Square, 1995.

17) A. D. McKale, B. W. Veal, A. P. Pailikas, C. K. Chan and G. S. Knapp: J. Am. Chem. Soc. 110 (1988) 3764. 